\title{
SPACES SATISFYING THE FIRST ENUMERABILITY AXIOM $\dagger$
}

\section{BY SELBY ROBINSON}

1. Introduction. A neighborhood of a point $p$ is a set of points to which $p$ is interior, $p$ being interior to a set $V$ if $p$ is a limit point of no subset of $C(V)$ and $V$ contains $p . \ddagger$ Using throughout the notation and concepts of Chittenden (loc. cit.), we define a topological space $(P, K)$ as a collection $P$ of points, and an undefined relation $K$ giving for each subset $E$ of $P$ a unique set $K(E)=E^{\prime}$ called the set of all limit points of $E$. Then $L(E)$ is the set of all limit points of all subsets of $E$; and $(P, L)$ a space formed from $(P, K)$ by taking as $E^{\prime}$ the set $L(E)$.

A space has property $D$ of Hausdorff if for any pair of points there are disjoined sets to which the points are respectively interior; and is regular if, instead of for every pair of points, the property holds for every pair of disjoined sets provided each contains all its $L$ points. Two families $F$ and $F^{\prime}$ of neighborhoods of a point are equivalent if each set of $F$ contains some set of $F^{\prime}$ and vice versa. For brevity we use $\left[U_{p}\right]$ consistently as a symbol for the family of all neighborhoods of the point $p$. The first enumerability axiom states that for each point $p$ there is an enumerable family of neighborhoods equivalent to $\left[U_{p}\right]$. As our definition of a space $V_{\omega}$, we adopt the one given by Fréchet in Espaces Abstraits, that is, a $V$ space in which for each point $p$ there is an enumerable decreasing family of neighborhoods whose product is $p$ and which is equivalent to $\left[U_{p}\right]$. Fréchet had in a previous definition required in addition that a space $V_{\omega}$ be an $L$ space. $\S$ By the fourth property of Riesz, $\uparrow$ we mean that

$\dagger$ Presented to the Society, April 3, 1931. The author is indebted to E. W. Chittenden for assistance in the preparation of this paper.

$\ddagger$ Fréchet, Esquisse d'une théorie des ensembles abstraits, Sir Asutosh Mookerjee's Commemoration volumes, Calcutta, 1922, vol. II, p. 362; Chittenden, Transactions of this Society, vol. 31 (1929), p. 296 and p. 293. By mistake the last clause was omitted from Chittenden's definition of interiority.

$\S$ See Les Espaces Abstraits, Paris, 1928, p. 216; and Transactions of this Society, vol. 19 (1918), p. 56.

ๆ Espaces Abstraits, pp. 209-10; D. McCoy, Tôhoku Mathematical Journal, 
for any set $E$ having any two points among its limit points, there is a subset $F$ of $E$ having as a limit point one but not the other of the two given points. An $L$ space is one having the first and third properties of Riesz and the property III': if $E^{\prime}>p$, there is a compact subset of $E$ having $p$ for a unique limit point. $\dagger$

2. Decreasing Families of Neighborhoods. If $p$ and $q$ are points of a topological space, a necessary and sufficient condition that $q$ be not in all neighborhoods of $p$ (or in all those of an equivalent family), is that $p$ be not in $q^{\prime}$. A necessary and sufficient condition that for every point $p$ the product of $\left[U_{p}\right]$ be $p$ is that no single point have a limit point outside itself.

Lemma 1. In a $V$ space, a necessary and sufficient condition that for each point $p$ the product of $\left[U_{p}\right]$ (or of any equivalent family) be $p$, is the third property of Riesz.

To prove that the third property of Riesz holds, we first notice that no point can have a limit point outside itself. In a $V$ space $p$ can be in $E^{\prime}$ only if in $(E-p)^{\prime}$. Let $E$ be $p+q$. Then $p$ cannot be in $E^{\prime}$, therefore not in $p^{\prime}$.

LeMma 2. A necessary condition that a point p have a decreasing family of neighborhoods equivalent to $\left[U_{p}\right]$ is that for any sets $B$ and $C$ such that $p<L(B+C), p$ is either in $L(B)$ or $L(C)$. Likewise, a necessary condition for every point to have the property is that the second property of Riesz hold in $(P, L)$. If in the first of these theorems the point $p$ have an enumerable family of neighborhoods equivalent to $\left[U_{p}\right]$, likerwise if, in the second the first enumerability axiom hold, the above conditions are also sufficient.

To prove the necessity, assume $p$ neither in $L(B)$ or $L(C)$. Then $P-B$ and $P-C$ are neighborhoods of $p$ and each contains a neighborhood of the decreasing family. The lesser of the two neighborhoods thus obtained is included in $P-(B+C)$. The proof of sufficiency is similar to that used in a theorem of

vol. 33 (1930), p. 90; Frink, this Bulletin, vol. 36 (1930), p. 282; Chittenden, loc. cit., p. 294. (The first $E$ in Chittenden's statement should be $E^{\prime}$.) See Riesz, Atti del 4 Congresso Internazionale dei Matematici, vol. 2, pp. 19-20; also Wiener, Bulletin de la Société Mathématique, vol. 50 (1922), pp. 128-129; and Fréchet, Bulletin des Sciences Mathématiques, (2), vol. 42, p. 139.

$\dagger$ See Espaces Abstraits, pp. 171-2 and 210-1; also Urysohn, L'Enseignement, vol. 25 (1926), pp. 77-82. 
Fréchet. $\dagger$ This part of the theorem (therefore part of Theorem 1 ) is really due to Chittenden, who proved to me that the second is the only one of the properties of Riesz used in obtaining a decreasing family.

TheOREM 1. If $(P, K)$ satisfies the first enumerability axiom, a necessary and sufficient condition for every point $p$ to have a decreasing family of neighborhoods (subfamily of the given enumerable family if desired) equivalent to $\left[U_{p}\right]$ and whose product is $p$ is that no point have a limit point outside itself and the second property of Riesz hold in $(P, L)$. Any point $p$ is either a neighborhood of itself or has an infinity of distinct neighborhoods in any family equivalent to $\left[U_{p}\right]$. If a particular point has an enumerable family of neighborhoods, the theorem holds for that point.

This follows from Lemma 2 and the first paragraph of this section. The fact that either $p$ is a neighborhood of itself or its family $F$ of neighborhoods is infinite, follows from the fact that the product of $F$ is $p$, and if $F$ is finite this product is a neighborhood. The sufficiency of the condition is a generalization of Fréchet's theorem cited above.

THEOREM 2. The validity of the second property of Riesz in $(P, K)$ implies its validity in $(P, L)$. The analogous property holds for a single point.

Suppose $p<L(B+C)$. Then $p<E^{\prime}$ for some $E<B+C$. By our hypothesis $p$ is either in $(E \cdot B)^{\prime}$ or $(E \cdot C)^{\prime}$, therefore either in $L(B)$ or $L(C)$. From this result we can get sufficient conditions to substitute for those of Lemma 2 and Theorem 1. From Lemma 2 and the equivalence of $L(E)$ and $E^{\prime}$ in a $V$ space, we get the following lemma.

Lemma 3. In a $V$ space satisfying the first enumerability axiom, a necessary and sufficient condition for the existence of the decreasing families of Lemma 2 is the second property of Riesz.

Theorem 3. A necessary and sufficient condition that a space be a space $V_{\omega}$ is that it satisfy the first three conditions of Riesz and the first enumerability axiom.

This follows immediately from Lemmas 1 and 3. I was led to

$\dagger$ American Journal of Mathematics, vol. 50 (1928), p. 62. 
investigate the necessity of this condition by a remark in a letter of Orrin Frink.

3. An Equivalence. Theorem 4. In a space $V_{\omega}$, the following properties are equivalent.

I. The fourth property of Riesz.

II. Property $D$ of Hausdorff.

III. The property of being an $L$ space.

In any space, III implies III', which implies I, so it suffices to prove that I implies II and II implies III. Suppose I holds but II is invalid. Then for some pair of points $p$ and $q$, if $V$ and $W$ are any neighborhoods of $p$ and $q$ respectively, $V \cdot W \neq 0$. As no point is isolated, there is an enumerably infinite decreasing sequence $V_{n}$ of distinct neighborhoods of $p$ equivalent to $\left[U_{p}\right]$, and a corresponding sequence $W_{n}$ for $q$. By omitting a finite number of neighborhoods at the beginning of each sequence, we can insure that $V_{n} \cdot q=0$ and that $W_{n} \cdot p=0$. Let $a_{n}$ be a point of $V_{n} \cdot W_{n}$ and let $A=\left[a_{n}\right]$. Then $p$ and $q$ are points of $A^{\prime}$ but not of $A$. By I there is a subset $B$ of $A$ which has $p$ (or $q$ ) but not $q$ for a limit point. But as any $W_{n}$ contains all but a finite number of the points of $A$, it contains a point of $B$. So $B^{\prime}>q$. To prove that II implies III, we consider a set $E$ having $p$ for a limit point, and by the use of a decreasing sequence of neighborhoods select from $E$ a compact subset having $p$ for a unique limit point.

The validity of II in a space $(P, K)$ satisfying all our hypotheses except the first property of Riesz is equivalent to the validity of I, II, or III in $(P, L)$. If property two of Riesz holds in $(P, L)$ (see Theorem 2), II implies I; and if the first enumerability axiom also holds, II implies III'.

Fréchet once stated that under our conditions I implies III. $\dagger$ Wiener published (loc. cit., pp. 131-2) an example which purported to invalidate this statement, remarking, however, that III holds if II also be assumed. Wiener's statement of the fourth postulate of Riesz, seemingly a translation of the one given by Fréchet in the article just cited, reads:

"If $A$ is a limit-element of $E$, and if $B$ is distinct from $A$, there is always at least one set $F$ which has $A$ for a limit-element with-

$\dagger$ Bulletin des Sciences Mathématiques, loc. cit., p. 149. 
out having $B$ for a limit-element." Wiener's example has this property if and only if $F$ is not required to be a subset of $E$. The fact that II is implied by I or III seems to be new, except for the known equivalence of II and III under the additional hypothesis of the closure of derived sets. $\dagger$

4. A Correction. Given any space $V_{\omega}$ containing a certain point $p$ not isolated, we construct from this a space $V_{\omega}$ not an $L$ space by adding an element $p^{*}$ which is a limit point of exactly the sets having $p$ for a limit point and which is such that for every $E, E^{\prime}=(E-p)^{\prime}$. Fréchet states that a space $V_{\omega}$ is necessarily an $L$ space and gives another sufficient condition which is also contradicted by our example; namely, that for any set $E$ having any point $q$ as a limit point, there is a sequence $N<E$ such that any neighborhood of $q$ contains all but a finite number of the points of $N$.

5. Questions of Enumerability. Sierpinski has stated and Putnam has generalized, certain equivalences involving the enumerability of increasing and decreasing families of closed sets. $\$$ All the theorems of these papers extend to topological space (substituting for the separability of a set $E$ the existence of an enumerable subset $N$ of $E$ such that $N+L(N)>E$, and for $E$ being clairsemé the non-existence of $M<E$ such that for any point $p$ in $M, L(M-p)>M)$; it being necessary, however, in those theorems for which Sierpinski assumed the closure of derived sets to assume that the interior of every set is open. From this we get a theorem which we state without proof.

$\dagger$ Hausdorff, Grundzüge der Mengenlehre, first edition, Leipzig, 1914, p. 263; Fréchet, American Journal of Mathematics, loc. cit., p. 65.

$\ddagger$ Espaces Abstraits, p. 216; Bulletin des Sciences Mathématiques, loc. cit., p. 147.

$\S$ Sierpinski, Fundamenta Mathematicae, vol. 2 (1921), p. 179; and Putnam, this Bulletin, vol. 36 (1930), p. 653. Putnam's statement that in any $V$ space in which derived sets are closed, Sierpinski's property II implies III and IV implies V, can be shown by an example to be incorrect. The correct statement is: $V$ spaces in which a set plus its derived set is closed. In the proof of both these theorems, it is the closure of the sets $F_{\alpha}=Q_{\alpha}+Q_{\alpha}^{\prime}$ which is needed, as the sets $Q_{\alpha}{ }^{\prime}$ are not known to be distinct. In Putnam's last theorem, separability should be replaced by the separability of every set. In $\$ 4$, p. 183 , Sierpinski does not succeed in proving the separability of every set, but merely the separability of the space. To remedy this, let the sequence (5) be, not all points of the space, but the points of some set assumed not separable. 
THEOREM 5. A space satisfies the first enumerability axiom if each point $p$ has a well ordered decreasing family of neighborhoods equivalent to $\left[U_{p}\right]$, the sets $L(E)$ are closed, and either every set is separable or the space is regular and every set is condensed in itself.

From this result we can get variations of Theorems 3 and 4 .

6. An Example. Frink proposed to me the problem (which he and Hildebrandt had considered) of constructing a bicompact separable, completely normal, Hausdorff space, satisfying the first enumerability axiom but not the second. The circumference of a circle is such a space if we define as follows the sets which for every point $p$ have $p$ as a limit point. Any set $E$ has $p$ as a limit point if and only if there are points of $E-p$ either (1), arbitrarily near $p$ in the direction clockwise from $p$, or (2), arbitrarily near $q$ (the point opposite $p$ ) in the direction counterclockwise from $q$. If we omit (2), we have a locally non-compact space having the other properties mentioned. $\dagger$

UNIVERSITY OF IOWA

\section{ADDENDUM ON FACTORABILITY OF NUMERICAL FUNCTIONS}

BY E. T. BELL

It has been pointed out to me that the statement of the Lemma on page 252 of the April, 1931, issue of this Bulletin might be misunderstood. To clear up any possible misunderstanding, it is sufficient to point out that the notation $f, g, h$ is as in the italicised statement on page 251 , as is evident on referring to paragraph 3 , third sentence. At the top of page 253, for "all or none of $f, g, \cdots, h$ are factorable," read "the only excluded possibility is that in which precisely one of $f, g, \cdots, h$ is not factorable." This is evident from paragraph 3 .

California Institute of Technology

$\dagger$ See Fréchet, Transactions of this Society, loc. cit., p. 60; and D. McCoy, loc. cit., p. 114 (S 241). 